Research Article

\title{
Prevalence and Antibiotic Resistance Patterns of Ocular Bacterial Strains Isolated from Pediatric Patients in University Hospital of Campania "Luigi Vanvitelli," Naples, Italy
}

\author{
Francesco Petrillo $\left(\mathbb{D},{ }^{1}\right.$ Veronica Folliero $\left(\mathbb{D},{ }^{2}\right.$ Biagio Santella $\left(\mathbb{D},{ }^{3}\right.$ Gianluigi Franci $\left(\mathbb{D},{ }^{4}\right.$ \\ Francesco Foglia $₫$, , Maria C. Trotta $\odot,{ }^{2}$ Maria T. Della Rocca $\odot{ }^{3},{ }^{3}$ Teresio Avitabile $\odot,{ }^{1}$ \\ Caterina Gagliano $\left(\mathbb{D},{ }^{5}\right.$ and Marilena Galdiero $\mathbb{i}^{2}$ \\ ${ }^{1}$ Section of Ophthalmology, University Hospital "Policlinic-Vittorio Emanuele", Catania, Italy \\ ${ }^{2}$ Department of Experimental Medicine, University of Campania "Luigi Vanvitelli", Naples, Italy \\ ${ }^{3}$ Section of Microbiology and Virology, University Hospital "Luigi Vanvitelli", Naples, Italy \\ ${ }^{4}$ Department of Medicine, Surgery and Dentistry "Scuola Medica Salernitana", University of Salerno, Baronissi, Italy \\ ${ }^{5}$ Section of Ocular Immunology and Rare Diseases, University Hospital "Policlinic-Vittorio Emanuele", Catania, Italy
}

Correspondence should be addressed to Marilena Galdiero; marilena.galdiero@unicampania.it

Received 7 May 2020; Revised 12 June 2020; Accepted 26 June 2020; Published 27 July 2020

Academic Editor: Joseph Falkinham

Copyright (c) 2020 Francesco Petrillo et al. This is an open access article distributed under the Creative Commons Attribution License, which permits unrestricted use, distribution, and reproduction in any medium, provided the original work is properly cited.

\begin{abstract}
Eye infections caused by bacteria are a serious public health problem among pediatric patients. These diseases, if not properly treated, can cause blindness and impaired vision. The study aimed to evaluate the antimicrobial resistance profiles of the main pathogens involved in eye infections. This study involved pediatric patients enrolled at the "Luigi Vanvitelli" University Hospital of Campania in Naples, Italy, between 2017 and 2019. Of a total of 228 pediatric patients, 73 (32\%) tested positive for bacterial infection. In terms of strain distribution, $85 \%$ were Gram-positive bacteria, while $15 \%$ were Gram-negative bacteria. The most frequently isolated strains were coagulase-negative Staphylococci (60.4\%), followed by Staphylococcus aureus (16.4\%). The isolated bacteria showed a significant percentage of resistance to multiple antibiotics. Therefore, the identification of the causal bacteria and antimicrobial sensitivity tests are mandatory to select the effective drug for the treatment of eye infections and prevent the development of antibiotic-resistant bacteria.
\end{abstract}

\section{Introduction}

Ocular infections and their complications represent an important public health problem [1]. These diseases are associated with a high degree of visual morbidity and blindness worldwide [2]. The ocular infections distribution in the population is conditional on many factors: (i) the use of contact lenses; (ii) surgery; (iii) trauma; (iv) previous eye infections; (v) obstruction of the nasolacrimal duct; (vi) age; and (vii) dry eye $[3,4]$. These infections are commonly observed in pediatric patients, affecting infants and preschool-aged children of both genders [5]. Bacteria are the main cause of ocular infection, although viruses, fungi, and parasites may be involved in the origin of this infection $[6,7]$. These microorganisms contribute to $32-74 \%$ of eye infections, globally [8]. Bacteria are associated with different types of eye surface infections including keratitis, dacryocystitis, blepharokeratoconjunctivitis, and conjunctivitis $[9,10]$. The most common bacterial pathogens, involved in pediatric ocular infection are coagulase-negative Staphylococci (CoNS), Staphylococcus aureus (S. aureus), Streptococcus pneumoniae, Pseudomonas aeruginosa ( $P$. aeruginosa), and Haemophilus influenzae [11]. Gram-positive bacteria are primarily responsible for pediatric ocular infection [12]. A prospective study conducted in the United States has revealed that $65 \%$ of children have ocular infections caused by Gram- 
positive bacteria [13]. According to the guidelines, the diagnosis of ocular bacterial infection is based on the examination of the patient's clinical symptoms and laboratory testing [14]. Cultural analysis and antibiotic susceptibility testing are ideal for guiding therapy. Although the guidelines for the treatment of these infections recommend the laboratory procedures, empirical broadspectrum antibiotics treatment is initially used [15]. This contributes to the development of antimicrobial resistance (AMR) among ocular pathogens, which has increased dramatically in recent decades [16]. A national surveillance study started in 2009 (ARMOR) has monitored the resistance profiles among bacterial species that most commonly cause eye infections: Staphylococci species, S. pneumoniae, $P$. aeruginosa, and $H$. influenzae. The study reported high rates of AMR, particularly among the Staphylococci species [17]. Ocular diseases, if not treated properly, can cause irreversible damage to the structures of the eye, leading to visual impairment and blindness [18]. Drug-resistant bacteria and the high prevalence of ocular bacterial infections in pediatric patients stress the importance of knowing the causative microorganisms and antimicrobials susceptibility profile. Therefore, the goal of our study was to evaluate the etiology and antimicrobial resistance profiles of ocular infection pathogens isolated from pediatric patients in the University Hospital of Campania "Luigi Vanvitelli," Naples.

\section{Materials and Methods}

2.1. Sample Collection. Our retrospective study was conducted on 228 pediatric patients with clinical diagnoses of ocular infections, at the University Hospital of Campania "Luigi Vanvitelli" (UOC) in Naples, Italy, between July 2017 and November 2019. Each patient had undergone a conjunctival sampling. This procedure consisted of rolling a thin cotton swab over the lower fornix of the conjunctival sac. The eye swab was inserted into the transport media and delivered to the bacteriology laboratory and processed.

2.2. Bacterial Culture and Identification. The samples were transferred into $5 \mathrm{ml}$ of Brain-Heart Infusion broth (Oxoid, Hampshire, UK) and incubated overnight at $37^{\circ} \mathrm{C}$. The broth was inoculated on blood agar, chocolate agar, MacConkey agar, mannitol salted agar, modified ThayerMartin agar, and Sabouraud glucose agar (Oxoid, Hampshire, United Kingdom). All plates were incubated overnight at $37^{\circ} \mathrm{C}$. The chocolate and Thayer-Martin agar were maintained in the presence of $\mathrm{CO}_{2}$. After 24 hours of incubation, each plate was examined, and negative plates were incubated for an additional 24 hours. Bacterial identification was obtained via Matrix-Assisted Laser Desorption Ionization-Time of Flight Mass Spectrometry (MALDI-TOF MS) (Bruker Dal-tonics, Germany). Identifications were performed according to the manufacturer's instructions. A score higher than 2 allowed a reliable identification of the species [19].
2.3. Antibiotic Susceptibility Test. Confirmation of identification obtained through MALDI-TOF MS and antibiotic sensitivity tests was performed through the Phoenix BD (Becton Dickinson, United States). Identifications and antibiotic sensitivity tests were performed according to the manufacturer's instructions. The examined antimicrobials in this study were: ampicillin, ciprofloxacin, gentamycin, fosfomycin, trimethoprim-sulfamethoxazole, fusidic acid, cefoxitin, ceftaroline, daptomycin, erythromycin, imipenem, linezolid, moxifloxacin, oxacillin, rifampicin, teicoplanin, tetracycline, tigecycline, mupirocin, vancomycin, nitrofurantoin, amikacin, amoxicillin-clavulanic acid, cefepime, cefotaxime, ceftazidime, cefuroxime, colistin, ertapenem, fosfomycin, meropenem, levofloxacin, piperacillin, piperacillin-tazobactam, tigecycline, and tobramycin ((Becton Dickinson, United States) [20]. Reference strains of Escherichia coli ATCC 25922 and S. aureus ATCC 25923 were used as quality control measure for identification criteria and antimicrobial susceptibility tests.

2.4. Data Analysis. Data were analyzed using IBM SPSS software (version 22.0; IBM SPSS Inc., New York, USA). Descriptive statistics were computerized for the study, and variables such as sex and pathogenic bacteria were isolated from the study population. The tables show the frequency of isolated ocular bacteria and also compare the resistance percentage of different antibiotics. For the categorical variables, the chi-square test values $<0.05$ were considered significant [21].

2.5. Ethical Consideration Statement. Ethical approval by the Human Research Ethics Committee was not requested for this study. The resignation was given as our study used laboratory management data and clinical information on patients, collected from databases. This is a retrospective study and not directly associated with patients. This study was consistent with the principles of the Helsinki Declaration.

\section{Results}

3.1. Incidence of Ocular Infections in Pediatric Patients. In this study, 228 ocular samples, obtained from pediatric patients, were processed. Ocular infections were diagnosed based on the patient's clinical symptoms, redness with mucopurulent discharge. As reported in Table 1, 32\% of patients were positive for bacterial growth, while $68 \%$ were negative (Table 1). Among the isolated strains, $85 \%$ was Gram-positive bacteria, while $15 \%$ was Gram-negative bacteria (Table 1). Our study showed a high frequency of ocular infections in males compared to females (31.5\%) (Table 1). In addition, we observed most cases over a 12month group (Figure 1).

Bacterial species, which appertain to 9 genera, were identified by 73 positive cultures. For Gram-positive bacteria, isolates of CoNS (Staphylococcus epidermidis, Staphylococcus haemolyticus, Staphylococcus warneri, Staphylococcus hominis, and Staphylococcus lugdunensis) were the most commonly 
TABLE 1: Ocular infection distribution of bacteria isolates among pediatric patients with gender and age.

\begin{tabular}{lc}
\hline Character & $n(\%)$ \\
\hline No bacterial growth & $155(68)$ \\
Bacterial growth & $73(32)$ \\
Gram+ & $62(85)$ \\
Gram- & $11(15)$ \\
Gender & \\
Female & $23(31.5)$ \\
Male & $50(68.5)$ \\
\hline
\end{tabular}

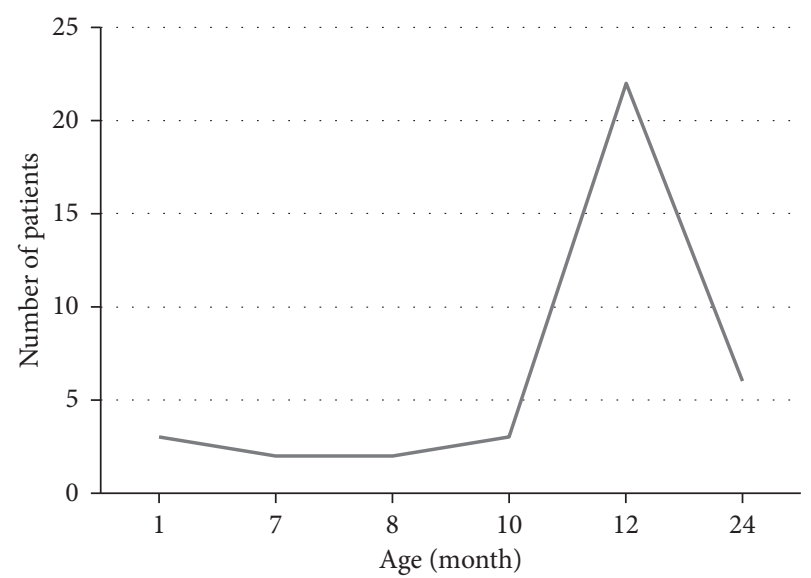

FIGURE 1: Frequency of age distribution in the pediatric patient's positive ocular swabs.

isolated bacteria, followed by S. aureus, Enterococcus species, Streptococcus salivarius, and Bacillus megatherium (Table 2). For Gram-negative bacteria, Enterobacter cloacae and Serratia marcescens have represented the bacteria frequently encountered, followed by Klebsiella pneumoniae, Escherichia coli, and Pseudomonas aeruginosa (Table 3).

3.2. Antimicrobial Resistance Profile of Bacterial Isolates. In this study, the antimicrobial resistant pattern of Grampositive and Gram-negative ocular bacterial isolates was evaluated. The most of Gram-positive isolates had shown greater resistance $(R>62,1 \%)$ to ampicillin, amoxicillinclavulanic acid, cefoxitin, erythromycin, and oxacillin. These strains had exhibited a higher rate of antibiotic sensitivity $(S>62.1 \%)$ to ceftaroline, daptomycin, linezolid, moxifloxacin, rifampicin, teicoplanin, tigecycline, mupirocin, vancomycin, trimethoprim-sulfamethoxazole, and nitrofurantoin (Table 4). Among the strains of S. aureus, 50\% were methicillin-resistant (MRSA) and had inducible macrolide Lincosamide-streptogramin B (MLSB) resistance phenotypes. Among the CoNS isolates, $72 \%$ were methicillin-resistant (MR-CoNS), and of these, only $41.4 \%$ had shown inducible MLSB resistance phenotypes.

The Gram-negative isolates had presented greater resistance $(R>63.6 \%)$ to ampicillin, cefepime, and cefuroxime. These strains had shown a high sensitivity $(S>63.6 \%)$ to amikacin, cefotaxime, ceftazidime, ciprofloxacin, colistin,
TABLE 2: Prevalence of Gram-positive bacteria isolated from ocular specimens.

\begin{tabular}{lc}
\hline \multicolumn{2}{c}{$\begin{array}{c}\text { Bacteria isolated from ocular specimens } \\
\text { Gram-positive bacteria }\end{array}$} \\
Isolates & $(\%)$ \\
\hline Staphylococcus aureus & 16.4 \\
CoNS & 60.4 \\
Enterococcus spp. & 5.4 \\
Streptococcus salivarius & 1.4 \\
Bacillus megatherium & 1.4 \\
\hline
\end{tabular}

Note: CoNS $^{*}=$ coagulase-negative Staphylococci.

TABle 3: Prevalence of Gram-negative bacteria isolated from ocular specimens.

\begin{tabular}{lc}
\hline \multicolumn{2}{c}{ Gram-negative bacteria } \\
Isolates & $(\%)$ \\
\hline \multicolumn{2}{c}{ Bacteria isolated from ocular specimens } \\
Klebsiella pneumoniae & 2.6 \\
Enterobacter cloacae & 4.1 \\
Serratia marcescens & 4.1 \\
Escherichia coli & 2.1 \\
Pseudomonas aeruginosa & 2.1 \\
\hline
\end{tabular}

TABLE 4: Sensitivity and resistance percentage of Gram-positive isolates.

\begin{tabular}{lcc}
\hline Antibiotics & Sensitivity (\%) & Resistance (\%) \\
\hline Ampicillin & & 100 \\
Amoxicillin-clavulanic acid & 58.6 & 100 \\
Fusidic acid & 36.2 & 41.4 \\
Cefoxitin & 62.1 & 63.8 \\
Ceftaroline & 91.4 & 37.9 \\
Daptomycin & 72.2 & 8.6 \\
Gentamicin & 36.2 & 27.8 \\
Erythromycin & 43.1 & 63.8 \\
Imipenem & 98.3 & 56.9 \\
Linezolid & 78 & 1.7 \\
Ciprofloxacin & 81 & 22 \\
Moxifloxacin & 37.9 & 19 \\
Oxacillin & 76.3 & 62.1 \\
Fosfomycin & 87.9 & 23.7 \\
Rifampicin & 91.4 & 12.1 \\
Teicoplanin & 60.3 & 39.7 \\
Tetracycline & 96.6 & 3.4 \\
Tigecycline & 94.9 & 5.1 \\
Mupirocin & 98.3 & 1.7 \\
Vancomycin & 76.3 & 23.7 \\
Trimethoprim-sulfamethoxazole & 91.5 & 8.5 \\
Nitrofurantoin & &
\end{tabular}

Note: $(-)=100 \%$ resistance.

ertapenem, gentamycin, meropenem, imipenem, levofloxacin, piperacillin-tazobactam, and trimethopim-sulfamethoxazolo (Table 5).

Resistance profile of the most represented bacterial isolates for common drugs of choice for empirical therapy is shown in Table 6. Bacterial strains most resistant to the common treatments of ocular infections were Enterococcus spp. for Gram-positive and K. pneumoniae for Gram-negative. 
TABLE 5: Sensitivity and resistance of Gram-negative isolates.

\begin{tabular}{lcc}
\hline Antibiotics & Sensitivity (\%) & Resistance (\%) \\
\hline Ampicillin & - & 100 \\
Amikacin & 63.6 & 36.4 \\
Amoxicillin-clavulanic acid & 18.2 & 36.4 \\
Cefepime & 36.4 & 63.6 \\
Cefotaxime & 72.7 & 27.3 \\
Ceftazidime & 72.7 & 27.3 \\
Cefuroxime & 9.1 & 90.9 \\
Ciprofloxacin & 72.7 & 27.3 \\
Colistin & 81.8 & 18.2 \\
Ertapenem & 72.7 & 27.3 \\
Fosfomycin & 54.5 & 45.5 \\
Gentamycin & 72.7 & 27.3 \\
Imipenem & 72.7 & 27.3 \\
Meropenem & 72.7 & 27.3 \\
Levofloxacin & 72.7 & 27.3 \\
Piperacillin & 54.5 & 45.5 \\
Piperacillin-tazobactam & 63.6 \\
Tigecycline & 36.4 \\
Tobramycin & 54.5 & 36.4 \\
Trimethoprim-sulfamethoxazole & 72.7 \\
\hline
\end{tabular}

TABle 6: Resistance rates of Gram-positive and Gram-negative isolates for common tested antibiotics.

\begin{tabular}{|c|c|c|c|c|c|c|c|c|}
\hline \multirow[b]{2}{*}{ Antibiotics (\%) } & \multicolumn{4}{|c|}{ Resistance profile for Gram-positive isolates } & \multicolumn{4}{|c|}{ Resistance profile for Gram-negative isolates } \\
\hline & S.aureus & CoNS & $\begin{array}{c}\text { Enterococcus } \\
\text { spp. }\end{array}$ & $\begin{array}{l}\text { Other Gram- } \\
\text { positive }\end{array}$ & $\begin{array}{c}\text { Klebsiella } \\
\text { pneumoniae }\end{array}$ & $\begin{array}{c}\text { Enterobacter } \\
\text { cloacae }\end{array}$ & $\begin{array}{c}\text { Serratia } \\
\text { marcescens }\end{array}$ & $\begin{array}{c}\text { Other Gram- } \\
\text { negative }\end{array}$ \\
\hline Ampicillin & 100 & 100 & 0 & 0 & 100 & 100 & 100 & 100 \\
\hline Ciprofloxacin & 14,3 & 24,4 & 0 & 0 & 66,7 & 0 & 0 & 60 \\
\hline $\begin{array}{l}\text { Amoxicillin-clavulanic } \\
\text { acid }\end{array}$ & 100 & 100 & 100 & 100 & 66,7 & 66,7 & 100 & 40 \\
\hline Gentamycin & 64,3 & 81,8 & 100 & 0 & 66,7 & 0 & 0 & 40 \\
\hline Fosfomycin & 7,1 & 24,4 & 100 & 0 & 66,7 & 33,3 & 66,7 & 20 \\
\hline $\begin{array}{l}\text { Trimethoprim- } \\
\text { sulphametroxazolo }\end{array}$ & 28,6 & 17,1 & 66,7 & 0 & 66,7 & 0 & 0 & 40 \\
\hline
\end{tabular}

\section{Discussion}

The bacterial ocular infections are commonly diagnosed in pediatric patients, affecting infants and preschool-aged children of both genders [22]. These diseases affect about 1 in every 8 children every year [23]. Although most cases are self-limiting, in others, about three weeks may be needed to remove the infection [24]. The gold standard for the treatment of bacterial ocular infection should be the identification of the agent and antibiotic susceptibility testing. In order to reduce antibiotic resistance, surveillance data of resistance profiles can guide the choice of appropriate empirical therapy in the absence of culture and sensitivity data [25]. The current analysis shows the incidence of ocular infections among pediatric patients, evaluating the pathogens involved in the infection and the related resistance profiles. In this study, 228 pediatric patients with supposed ocular surface infection were enrolled. Of these, $32 \%$ were suffering from an ocular infection. Similar proportions had been observed in India (34.5\%), Japan (32.2\%), and Iran (37.5\%) [26-28]. A higher incidence was recorded in Ethiopia (74.7\%) and Jordan (54.2\%) [29, 30].
Sociodemographic and geographic aspects could explain these differences [31]. The high frequency of ocular infections was observed in the 12-month group. The elevated prevalence in this age group is mainly due to poor hand hygiene [32]. It was interesting to note that males $(68.5 \%)$ were more susceptible to ocular infections, contrary to the study of Teweldemedhin et al. (Ethiopia) [8], where the females represented $55.9 \%$. In another study conducted in Iran, there was no significant difference in the incidence of ocular infection among male and female patients [33]. This variation in the gender rate can differ from country to country. As reported in India, Iran, Ethiopia, and Jordan, Gram-positive bacteria were mainly responsible for bacterial ocular infections among pediatric patients [34]. In our study, CoNS were the most isolated strains (60.4\%), and according to the study of Muluye et al., although CoNS constitute the normal flora of the skin and their presence could be due to contamination during sampling, we believe that they represent a source of infection as they are associated with clinical symptoms [35]. In a retrospective study conducted in India, CoNS had caused $45.4 \%$ of ocular infections [36]. Similar data were reported in Iran with a prevalence of $40 \%$. 
Gram-negative bacteria only partially contribute to ocular infections, according to the study of Mohammad [37]. The most isolated strains were Enterobacter cloacae (4.1\%) and Serratia marcescens (4.1\%). Different results were observed in Ethiopia, where the most isolated Gram-negative strain is Pseudomonas aeruginosa (11.3\%). Among Gram-positive bacteria, ampicillin, amoxicillin-clavulanic acid, cefoxitin, erythromycin, and oxacillin showed a lower performance, recording resistance rates greater than $63.8 \%$. daptomycin, linezolid, teicoplanin, tigecycline, moxifloxacin, rifampicin, mupirocin, rifampicin, teicoplanin, tigecycline, vancomycin, and nitrofurantoin had exhibited a higher efficacy against Gram-positive strains $(S>81 \%)$. Methicillin resistance was detected in $72 \%$ of the isolated CoNS. A lower incidence of these strains was observed in the United States (47.4\%), in Uganda (27.6\%), and in Ethiopia (45.2\%). Concerning Gram-negative bacteria, all strains had shown a high rate of resistance to ampicillin and cefuroxime $(R>90.9 \%)$. They had exhibited high efficacy of cefotaxime, ceftazidime, colistin, ertapenem, meropenem, imipenem, gentamycin, levofloxacin, and trimethoprim-sulfamethoxazole $(R>72.7 \%)$. However, fluoroquinolones represent the most used antibiotics in ophthalmic practice, and they remain effective against the strains responsible for ocular infections [38]. The Gramnegative and -positive isolated bacteria in our University Hospital had shown a low rate of resistance to the fluoroquinolones tested (ciprofloxacin, moxifloxacin, and levofloxacin). Antibiotics, belonging to this class, inhibit DNA gyrase and topoisomerase IV, enzymes that are involved in DNA replication [39]. These drugs are broad-spectrum antibiotics, providing excellent coverage against most ocular pathogens [40]. It is well tolerated on the ocular surface, and the topical use reduces the development of bacterial resistance [41].

These data can be the starting point for outlining the guideline in the treatment of the pediatric patient's ocular infection. In conclusion, the main goal of our study was to report the bacterial profile and antibiotic susceptibility pattern of ocular infection in pediatric patients in order to know the epidemiology of our hospital, reducing the antibiotic resistance and improving the empirical treatment with factual and statistical information.

\section{Data Availability}

Epidemiological data used to support the results of this study are included in the article.

\section{Conflicts of Interest}

None of the authors have any conflicts of interest related to this submission.

\section{Acknowledgments}

The authors would like to thank the staff of the U.O.C University Hospital of Campania "Luigi Vanvitelli" in Naples, Italy, for their contributions.

\section{References}

[1] S. Watson, M. Cabrera-Aguas, and P. Khoo, "Common eye infections," Australian Prescriber, vol. 41, no. 3, pp. 67-72, 2018.

[2] M. V. Prakash, S. Sivakumar, A. Dayal, A. Chitra, and S. Subramaniam, "Ocular morbidity patterns among children in schools for the blind in Chennai," Indian Journal of Ophthalmology, vol. 65, no. 8, pp. 733-737, 2017.

[3] M. D. P. Willcox, "Pseudomonas aeruginosa infection and inflammation during contact lens wear: a review," Optometry and Vision Science, vol. 84, no. 4, pp. 273-278, 2007.

[4] A. Grzybowski, P. Brona, and S. J. Kim, "Microbial flora and resistance in ophthalmology: a review," Graefe's Archive for Clinical and Experimental Ophthalmology, vol. 255, no. 5, pp. 851-862, 2017.

[5] A. Bates, "Common eye problems among children," London Journal of Primary Care, vol. 3, no. 1, pp. 27-30, 2010.

[6] S. Xu and L. D. Hazlett, "MicroRNAs in ocular infection," Microorganisms, vol. 7, no. 9, p. 359, 2019.

[7] M. Teweldemedhin, H. Gebreyesus, A. H. Atsbaha, S. W. Asgedom, and M. Saravanan, "Bacterial profile of ocular infections: a systematic review," BMC Ophthalmology, vol. 17, no. 1, p. 212, 2017.

[8] M. Teweldemedhin, M. Saravanan, A. Gebreyesus, and D. Gebreegziabiher, "Ocular bacterial infections at Quiha Ophthalmic Hospital, Northern Ethiopia: an evaluation according to the risk factors and the antimicrobial susceptibility of bacterial isolates," BMC Infectious Diseases, vol. 17, no. 1, p. 207, 2017.

[9] R. P. Kowalski, S. V. Nayyar, E. G. Romanowski et al., "The prevalence of bacteria, fungi, viruses, and Acanthamoeba from 3,004 cases of keratitis, endophthalmitis, and conjunctivitis," Eye Contact Lens: Science \& Clinical Practice, vol. 2019, 2019.

[10] X. Song, L. Xu, S. Sun, J. Zhao, and L. Xie, "Pediatric microbial keratitis: a tertiary hospital study," European Journal of Ophthalmology, vol. 22, no. 2, pp. 136-141, 2012.

[11] E. Getahun, B. Gelaw, A. Assefa, Y. Assefa, and A. Amsalu, "Bacterial pathogens associated with external ocular infections alongside eminent proportion of multidrug resistant isolates at the University of Gondar Hospital, northwest Ethiopia," BMC Ophthalmology, vol. 17, no. 1, p. 151, 2017.

[12] G. Grandi, G. Bianco, M. Boattini et al., "Bacterial etiology and antimicrobial resistance trends in ocular infections: a 30-year study, Turin area, Italy," European Journal of Ophthalmology, vol. 2019, 2019.

[13] E. Mantadakis, S. Maraki, L. Michailidis, Z. Gitti, I. G. Pallikaris, and G. Samonis, "Antimicrobial susceptibility of Gram-positive cocci isolated from patients with conjunctivitis and keratitis in Crete, Greece," Journal of Microbiology, Immunology and Infection, vol. 46, no. 1, pp. 41-47, 2013.

[14] S. Sharma, "Diagnosis of infectious diseases of the eye," Eye, vol. 26, no. 2, pp. 177-184, 2012.

[15] A. Austin, J. Schallhorn, M. Geske, M. Mannis, T. Lietman, and J. Rose-Nussbaumer, "Empirical treatment of bacterial keratitis: an international survey of corneal specialists," BMJ Open Ophthalmology, vol. 2, no. 1, Article ID e000047, 2017.

[16] S. Sharma, "Antibiotic resistance in ocular bacterial pathogens," Indian Journal of Medical Microbiology, vol. 29, no. 3, pp. 218-222, 2011.

[17] K. T. Randall, R. Melton, and P. A. Asbell, "Antibiotic resistance among ocular pathogens: current trends from the 
ARMOR surveillance study (2009-2016)," Clinical Optometry (Auckl), vol. 11, pp. 15-26, 2019.

[18] S. W. Teh, P. L. Mok, A. Rashid et al., "Recent updates on treatment of ocular microbial infections by stem cell therapy: a review," International Journal of Molecular Sciences, vol. 19, no. 2, p. 558, 2018.

[19] Y. Yang, Y. Lin, and L. Qiao, "Direct MALDI-TOF MS identification of bacterial mixtures," Analytical Chemistry, vol. 90, no. 17, pp. 10400-10408, 2018.

[20] V. Folliero, P. Caputo, M. T. D. Rocca et al., "Prevalence and antimicrobial susceptibility patterns of bacterial pathogens in urinary tract infections in university hospital of Campania "luigi Vanvitelli" between 2017 and 2018," Antibiotics (Basel), vol. 9 , no. 5, p. 215, 2020.

[21] M. A. Gouda, "Common pitfalls in reporting the use of SPSS software," Medical Principles and Practice, vol. 24, no. 3, p. 300, 2015.

[22] A. K. C. Leung, K. L. Hon, A. H. C. Wong, and A. S. Wong, "Bacterial conjunctivitis in childhood: etiology, clinical manifestations, diagnosis, and management," Recent Patents on Inflammation \& Allergy Drug Discovery, vol. 12, no. 2, pp. 120-127, 2018.

[23] R. Chawla, J. D. Kellner, and W. F. Astle, "Acute infectious conjunctivitis in childhood," Paediatrics \& Child Health, vol. 6 , no. 6, pp. 329-335, 2001.

[24] A. A. Azari and N. P. Barney, "Conjunctivitis," JAMA, vol. 310, no. 16, pp. 1721-1729, 2013.

[25] D. Muluye, Y. Wondimeneh, F. Moges, T. Nega, and G. Ferede, "Types and drug susceptibility patterns of bacterial isolates from eye discharge samples at Gondar University Hospital, Northwest Ethiopia," BMC Research Notes, vol. 7, no. 1, p. 292, 2014.

[26] S. Ramesh, R. Ramakrishnan, M. J. Bharathi, V. Amuthan, and S. Visanathan, "Prevalence of bacterial pathogens causing ocular infections in South India," Indian Journal of Pathology Microbiology, vol. 53, no. 2, pp. 281-286, 2010.

[27] Y. Shimizu, H. Toshida, R Honda et al., "Prevalence of drug resistance and culture-positive rate among microorganisms isolated from patients with ocular infections over a 4-year period," Clinical Ophthalmology (Auckland, N.Z.), vol. 7, pp. 695-702, 2013.

[28] M. M. J. Moghadam, M. M. Yari, F. A. Jalilian, R. Amini, and N. Bazzazi, "Epidemiology and molecular diagnosis of acute conjunctivitis in patients attending Hamadan, west Iran ophthalmology clinics 2016-2017," Clinical Optometry (Auckl), vol. 11, pp. 105-111, 2019.

[29] M. Chaudhary, A. Bhattarai, S. Adhikari, and D. Bhatta, "Bacteriology and antimicrobial susceptibility of adult chronic dacryocystitis," Nepalese Journal of Ophthalmology, vol. 2, no. 2, pp. 105-113, 2010.

[30] J. P. Deibel and K. Cowling, "Ocular inflammation and infection," Emergency Medicine Clinics of North America, vol. 31, no. 2, pp. 387-397, 2013.

[31] F. V. Chen, T. C. Chang, and K. M. Cavuoto, "Patient demographic and microbiology trends in bacterial conjunctivitis in children," Journal of American Association for Pediatric Ophthalmology and Strabismus, vol. 22, no. 1, pp. 66-67, 2018.

[32] R. Mearkle, R. Houghton, D. Bwonya, and R. Lindfield, "Barriers to hand hygiene in ophthalmic outpatients in Uganda: a mixed methods approach," Journal of Ophthalmic Inflammation and Infection, vol. 6, no. 1, p. 11, 2016.

[33] P. Courtright and S. K. West, "Contribution of sex-linked biology and gender roles to disparities with Trachomal,"
Emerging Infectious Diseases, vol. 10, no. 11, pp. 2012-2016, 2004.

[34] A. Okesola and A. Okesola, "Microbiological profile of bacterial conjunctivitis in Ibadan, Nigeria," Annals of Ibadan Postgraduate Medicine, vol. 8, no. 1, pp. 20-24, 2010.

[35] K. Becker, C. Heilmann, and G. Peters, "Coagulase-negative staphylococci," Clinical Microbiology Reviews, vol. 27, no. 4, pp. 870-926, 2014.

[36] H. Deguchi, K. Kitazawa, K. Kayukawa et al., "The trend of resistance to antibiotics for ocular infection of Staphylococcus Aureus, Coagulase-Negative Staphylococci, and Corynebacterium compared with 10-years previous: a retrospective observational study," PLoS One, vol. 13, no. 9, Article ID e0203705, 2018.

[37] A.-S. Mohammad, "Etiology and antibacterial susceptibility pattern of bacterial ocular infections in a children hospital in north Jordan (2005-2009)," Biomedical \& Pharmacology Journal, vol. 5, no. 1, pp. 25-31, 2012.

[38] J. S. Bertino, "Impact of antibiotic resistance in the management of ocular infections: the role of current and future antibiotics," Clinical Ophthalmology, vol. 3, pp. 507-521, 2009.

[39] G. Franci, V. Folliero, M. Cammarota et al., "Epigenetic modulator UVI5008 inhibits MRSA by interfering with bacterial gyrase," Scientific Reports, vol. 8, no. 1, p. 13117, 2018.

[40] N. Wang, Q. Huang, Y. Tan, L. Lin, and K. Wu, "Bacterial spectrum and resistance patterns in corneal infections at a tertiary eye care center in south China," International Journal of Ophthalmology, vol. 9, no. 3, pp. 384-389, 2016.

[41] J. Epling, "Bacterial conjunctivitis," BMJ Clinical Evidence, vol. 2012, p. 0704, 2012. 